vs. $23 \mathrm{~min}, \mathrm{p}=0.64)$. The median number of passes required for recanalization was lower in the direct aspiration group (1 vs. $2, \mathrm{p}=0.01)$. Although there was no difference in successful recanalization (TICI $2 \mathrm{~b}-3)$ between the groups $(86.1 \%$ vs. $88 \%, \mathrm{p}=0.71)$, there was a lower rate of complete recanalization (TICI 2c-3) in the direct aspiration group (46\% vs. $51.7 \%, \mathrm{p}=0.007)$. There was also a lower rate of adjunctive treatments (defined as the use of GP IIb/IIIa inhibitors, P2Y12 inhibitors, and/or salvage angioplasty and/or stenting) required in the direct contact aspiration group $(36.1 \%$ vs. $44.4 \%, \mathrm{p}<$ $0.001)$. There were no differences in discharge NIHSS scores $(5$ vs. $4, \mathrm{p}=0.21)$ or in-hospital mortality $(22.2 \%$ vs. $22.5 \%, \mathrm{p}=0.92)$.

Conclusions In the NVQI-QOD registry, stent-retriever techniques were associated with higher rates of complete recanalization when compared to direct contact aspiration alone, but successful (TICI 2b-3) recanalization rates were similar. There were no statistically significant differences in procedure times, clinical outcomes at discharge, or in-hospital mortality.

Disclosures A. Mahapatra: None. Y. Moazeni: None. T. Patterson: None. R. Abdalla: None. J. Tsai: None. N. Moore: None. M. Bain: None. G. Toth: None. S. Ansari: None. M. Hussain: None.

\section{P-011 RESCUE OF NEGLECT AND LANGUAGE IMPAIRMENT AFTER STROKE THROMBECTOMY}

${ }^{1} \mathrm{~S}$ Desai ${ }^{*},{ }^{2} \mathrm{~K}$ Malhotra, ${ }^{1} \mathrm{G}$ Ramaiah, ${ }^{1} \mathrm{D}$ Tonetti, ${ }^{1} \mathrm{~W}$ Huq, ${ }^{3} \mathrm{~T}$ Jovin, ${ }^{1} \mathrm{~A}$ Jadhav. ${ }^{1}$ University of Pittsburgh Medical Center, Pittsburgh, PA; ${ }^{2}$ Allegheny Health Network, Pittsburgh, PA; ${ }^{3}$ Cooper University, Camden, NJ

\subsection{6/neurintsurg-2021-SNIS.47}

Introduction Although National Institutes of Health Stroke Scale (NIHSS) scores provide an objective measure of clinical deficits, data regarding the impact of neglect or language impairment on outcomes after mechanical thrombectomy (MT) is lacking. We assessed the frequency of neglect and language impairment, rate of their rescue by MT, and impact of rescue on clinical outcomes.

Methods This is a retrospective analysis of a prospectively collected database from a comprehensive stroke center. We assessed right (RHS) and left hemispheric strokes (LHS) patients with anterior circulation large vessel occlusion (ACLVO) undergoing MT to assess the impact of neglect and language impairment on clinical outcomes, respectively. Safety and efficacy outcomes were compared between patients with and without rescue of neglect or language impairment.

Results Among 324 RHS and 210 LHS patients, 71\% of patients presented with neglect whereas $93 \%$ of patients had

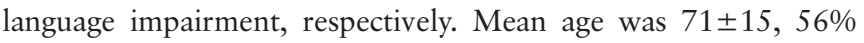
were females, and median NIHSS was 16 (12-20). At 24 hours, MT resulted in rescue of neglect in 31\% of RHS and rescue of language impairment in $23 \%$ of LHS patients, respectively. RHS patients with rescue of neglect $(56 \%$ vs $34 \%, \mathrm{p}<0.001)$ and LHS patients with rescue of language impairment $(64 \%$ vs $25 \%, \mathrm{p}<0.01)$ were observed to have a higher rate of functional independence compared to patients without rescue. After adjusting for confounders including 24hour NIHSS, rescue of neglect among RHS patients was associated with functional independence $[p=0.01]$ and lower mortality $[p=0.01]$. Similarly, rescue of language impairment among LHS patients was associated with functional independence $[p=0.02]$ and lower mortality $[p=0.001]$.
Conclusion Majority of LHS-ACLVO and of RHS-ACLVO patients present with the impairment of language and neglect, respectively. In comparison to 24-hour NIHSS, rescue of these deficits by MT is an independent and a better predictor of functional independence and lower mortality.

Disclosures S. Desai: None. K. Malhotra: None. G. Ramaiah: None. D. Tonetti: None. W. Huq: None. T. Jovin: None. A. Jadhav: None.

\section{P-012 THROMBECTOMY ASPIRATION POST-MARKET STUDY IN ACUTE STROKE: THE TAPAS STUDY}

${ }^{1} \mathrm{~F}$ Ballenilla* ${ }^{2} \mathrm{M}$ Espinosa, ${ }^{2} \mathrm{~J}$ Zamarro, ${ }^{3} \mathrm{~J}$ Masso, ${ }^{3} \mathrm{E}$ Garmendia, ${ }^{4} \mathrm{~J}$ Pumar, ${ }^{3} \mathrm{~J}$ Larrea, ${ }^{5}$ A Mosqueira. ${ }^{1}$ Hospital Universitario 12 De Octubre, Madrid, Spain; ${ }^{2}$ Hospital Virgen de la Arrixaca, Murcia, Spain; ${ }^{3}$ Centro Hospitalario Universitario Donostia, San Sebastian, Spain; ${ }^{4} 4$ Hospital Universitario de Santiago de Compostela, Santiago de Compostela, Spain; ${ }^{5}$ Hospital Universitario de Santiago de Compostela, Santiago de Compostela, Spain

\subsection{6/neurintsurg-2021-SNIS.48}

Introduction The Q Aspiration Catheter offers a novel design in which the proximal three-quarters of the catheter shaft is replaced with a pusher wire and the aspiration source is attached directly to the $8 \mathrm{~F}$ guide catheter. This design results in an increased aspiration force due to the larger $8 \mathrm{~F}$ guide catheter lumen. This first clinical study was designed to provide initial safety and performance data on the $\mathrm{Q}$ Aspiration Catheter. The study was a multi-center, observational, post market study utilizing the Q Catheter as first-line therapy during mechanical thrombectomy for acute ischemic stroke in Spain.

Methods Patients with AIS treated with MT using the Q Aspiration Catheter as first line therapy between March 2019 and January 2020 were identified at four high volume centers in Spain. Patients 18-85 years of age who had anterior or posterior LVO with an ASPECTs score 6-10 and were within 8 hours of onset/last known well were eligible to participate. Baseline demographics, procedural data, post procedure neuroimaging, and clinical outcomes were reported. A follow up visit was conducted to assess mRS.

Results A total of 45 patients provided written informed consent. Average patient age at presentation was 72.4 (range 4984 ) and $53.3 \%$ were male. Thrombolytic therapy was given in $46.7 \%$ (21/45) and NIHSS was 14.4 (range 1-26) at presentation. Occlusion site was $42(94 \%)$ in the anterior circulation: ICA-T in 9 patients (20\%), M1 MCA in 25 (56\%), and M2 MCA in $8(18 \%)$. Occlusion site in the posterior circulation was $3(6 \%)$. The Q Catheter was successfully delivered to the occlusion $89 \%(40 / 45)$ of the time, successful delivery increased to $96 \%(43 / 45)$ when including a stent retriever for anchoring. Successful revascularization of final mTICI $2 b-3$ was achieved in 93\% of patients (42/45). First pass success of mTICI 2b-3 with only a Q Catheter aspiration was achieved in $49 \%(21 / 43)$, this increased to $56 \%(24 / 43)$ when a stent retriever was used as an anchor. Overall successful revascularization using the $\mathrm{Q}$ Catheter, alone or with a stent retriever, was 77\% (33/43). Three patients required angioplasty and an implantable stent due to intracranial stenosis of atherosclerotic plaque. ENT occurred in 2\% (1), sICH rate was $2 \%(1)$ and not related to the device or procedure, mortality during the study period was $13 \%$ (6). During the study period, $55.5 \%$ of the subjects had a Modified Rankin score of 0-2 at follow up. Conclusion The Q Aspiration Catheter is a novel technology that showed comparable deliverability and revascularization 